\title{
Monitoramento automático da qualidade das informações que contribuem para a transparência pública
}

\author{
Fabricio Massariol, Fabiano da Rocha Louzada \\ Secretaria de Estado de Controle e Transparência do ES \\ Av. Governador Bley, 236, Centro - Vitória/ES - Brasil \\ \{fabricio.massariol, Fabiano.louzada\}@secont.es.gov.br
}

\begin{abstract}
With the application of the Law on Access to Public Information, in 2012, transparency portals have been expanded to comply with this legislation. However, not all public managers have sufficient resources to analyze the information that is being uploaded daily. Providing information without quality will not meet the basic principle of transparency, that of making society understand information and participating in government actions. This article demonstrates how to operationalize a data analysis of a transparency portal and how this operation to improve the quality characteristics of the information to the public.

Resumo. Com a vigência da Lei de Acesso à Informação, em 2012, os portais de transparência têm sido ampliados para atendimento a esta legislação. Contudo, nem todos os gestores públicos possuem recursos suficientes para analisar as informações que estão sendo pulicadas diariamente. Disponibilizar a informação sem qualidade não atenderá ao princípio básico da transparência, o de fazer com que a sociedade entenda as informações e participe dos atos governamentais. Este artigo demonstra como operacionalizar a análise dos dados de um portal da transparência e como esta operação contribui para melhorar as características de qualidade das informações disponíveis ao público.
\end{abstract}

\section{Introdução}

É notório o avanço no uso de novas tecnologias para disponibilização de informações à sociedade, porém, a credibilidade nas informações disponibilizadas pelos portais de dados abertos e de transparência ainda é um grande desafio, principalmente em países em desenvolvimento, conforme destacado por Dabla-Norris et al. (2010) e Gollwitzer (2010).

Parte desse problema é devido à heterogeneidade das informações publicadas, sua forma de atualização e frequência. A Modernização e Ampliação do Portal da Transparência do Governo do Estado do Espírito Santo [Massariol, Louzada and Alves, 2017] demonstra que para atendimento à Lei de Responsabilidade Fiscal [Brasil, 2000] e Lei de Acesso à Informação [Brasil, 2011], foi necessário buscar dados em diversos sistemas da administração pública, alguns provenientes de bancos de dados, integrados de forma automática e atualizados diariamente, outros, produzidos em planilhas e atualizados manualmente pelos respectivos responsáveis.

Manter dados e informações disponíveis aos cidadãos, provenientes de vários sistemas, com frequência de atualização e integrações específicas, de forma íntegra, correta 
e atualizada, exige um esforço grande de validações por parte do gestor público que, muitas vezes, não possui recursos suficientes para realizar tal procedimento.

Este artigo irá demonstrar como operacionalizar a análise dos dados de um portal da transparência, por um web crawler, para atendimento às características de qualidade da informação. Para tanto, será usado o robô MAILA (acrônimo de Monitoramento Automático de Informações de Livre Acesso), utilizado no Portal da Transparência do Governo do Estado do Espírito Santo, e os trabalhos desenvolvidos por Parker et al. (2006) e Cappelli (2009), que fornecem um conjunto de características que contribuem para a transparência das informações.

Segundo Reis (2013), um web crawler é um programa de computador que busca e recupera informações da Web de forma automática, "visitando" suas páginas e armazenando as informações em um repositório central para que sejam analisadas para diversos propósitos, por exemplo, para suportar um mecanismo de busca geral, um mecanismo de busca especializado em um determinado assunto, para análises de inteligência de negócios (coletar informações sobre empresas concorrentes, parceiros de negócio, notícias relevantes), para o monitoramento de web sites de interesse, etc.

Na seção 2 é apresentada a fundamentação teórica; na seção 3, as características e funcionamento do robô MAILA; na seção 4, as características da qualidade da informação verificadas; na seção 5, seus benefícios e resultados obtidos até o momento; na seção 6 , conclusão e perspectivas futuras.

\section{Fundamentação Teórica}

O primeiro sítio eletrônico designado de Portal da Transparência foi elaborado em 2004, pela Controladoria Geral da União (CGU) (2018), com a finalidade de publicar informações orçamentárias e financeiras, conforme estabelece a Lei de Responsabilidade Fiscal - LRF [Brasil, 2000]. O Estado do Espírito Santo elaborou e publicou seu portal em 2009, sob a responsabilidade da Secretaria de Estado de Controle e Transparência, de acordo com os prazos estabelecidos pela Lei Complementar $n^{\circ}$ 131/2009 [Brasil, 2009], que determina a disponibilização, em tempo real, de informações pormenorizadas sobre a execução orçamentária e financeira da União, dos Estados, do Distrito Federal e dos Municípios.

No ano de 2011 foi publicada a Lei de Acesso à Informação - LAI, nº 12.527/2011 [Brasil, 2011], que estabeleceu a diretriz que todos os documentos são públicos e, o sigilo, a exceção. Neste momento, o rol taxativo de informações descritas pela LRF, passou a ser o rol mínimo exigido pela LAI, além da exigência em ter um portal amigável e que pudesse alcançar o maior número possível de cidadãos. Os desafios impostos por esta nova legislação, levou o Governo do Estado do Espírito Santo a elaborar um projeto de modernização e ampliação do Portal da Transparência, com informações e funcionalidades bem mapeadas e que pudessem abranger os três perfis de públicos-alvo mapeados pela pesquisa [Massariol, Louzada and Alves, 2017].

O resultado do trabalho foi a ampliação do número de consultas disponíveis, provenientes de vários sistemas do Poder Executivo e do Tribunal de Contas, com diversas formas de integração e frequências de atualização, conforme listado no Quadro 1 a seguir:

\begin{tabular}{|l|l|l|l|l|}
\hline Assunto & Responsável & Sigla do & Origem dos dados / & Frequên \\
\hline
\end{tabular}




\begin{tabular}{|l|l|l|l|l|}
\hline & & Sistema & Ferramenta de integração & cia \\
\hline $\begin{array}{l}\text { Despesa e } \\
\text { receitas }\end{array}$ & Secretaria da Fazenda & SIGEFES & Arquivos CSV / Hang fire & Diária \\
\hline $\begin{array}{l}\text { Licitações e } \\
\text { Contratos }\end{array}$ & $\begin{array}{l}\text { Secretaria de Recursos } \\
\text { Humanos }\end{array}$ & SIGA & $\begin{array}{l}\text { Banco SQL-SERVER / } \\
\text { Integration Server }\end{array}$ & Diária \\
\hline Obras & Tribunal de Contas & Geo-Obras & Banco Oracle / Hang Fire & Diária \\
\hline $\begin{array}{l}\text { Leis } \\
\text { orçamentárias }\end{array}$ & $\begin{array}{l}\text { Secretaria de } \\
\text { Planejamento }\end{array}$ & - & $\begin{array}{l}\text { Sítio institucional / Web } \\
\text { Service }\end{array}$ & Anual \\
\hline $\begin{array}{l}\text { Convênios e } \\
\text { Transferências }\end{array}$ & Secretaria da Fazenda & SIGEFES & Arquivos CSV / Hang Fire & Diário e \\
\hline $\begin{array}{l}\text { Cartão } \\
\text { corporativo }\end{array}$ & $\begin{array}{l}\text { Secretaria de Recursos } \\
\text { Humanos }\end{array}$ & - & Arquivos CSV / Hang fire & Mensal \\
\hline $\begin{array}{l}\text { Bolsa } \\
\text { Capixaba }\end{array}$ & $\begin{array}{l}\text { Secretaria de Assistência } \\
\text { Social }\end{array}$ & $\begin{array}{l}\text { Sistema da } \\
\text { CEF }\end{array}$ & Arquivos CSV / Hang fire & Mensal \\
\hline Patrimônio & $\begin{array}{l}\text { Secretaria de Recursos } \\
\text { Humanos }\end{array}$ & SIGA & $\begin{array}{l}\text { Banco SQL-SERVER / } \\
\text { Integration Server }\end{array}$ & Diária \\
\hline $\begin{array}{l}\text { Servidores } \\
\text { Públicos }\end{array}$ & $\begin{array}{l}\text { Secretaria de Recursos } \\
\text { Humanos }\end{array}$ & SIARHES & Banco Oracle / Hang Fire & Diária \\
\hline $\begin{array}{l}\text { Prestação de } \\
\text { Contas do } \\
\text { Governador }\end{array}$ & $\begin{array}{l}\text { Secretaria de Controle e } \\
\text { Transparência e Tribunal } \\
\text { de Contas }\end{array}$ & - & Arquivos em PDF & Anual \\
\hline Saúde & Secretaria de Saúde & $\begin{array}{l}\text { Sistema de } \\
\text { indicadores }\end{array}$ & Arquivo XML / Web Service & Diária \\
\hline Segurança & $\begin{array}{l}\text { Secretaria de Segurança } \\
\text { Pública }\end{array}$ & SIAT & Banco Oracle / Hang Fire & Diária \\
\hline Educação & Secretaria de Educação & SIGES & WebService / Hang Fire & Semanal \\
\hline Conselhos & Secretaria de Governo & - & Planilha excel / atualizado \\
pelo Usuário & mensal \\
\hline Mapas & $\begin{array}{l}\text { Instituto Capixaba de } \\
\text { Técnica e Extensão Rural }\end{array}$ & GeoBases & $\begin{array}{l}\text { View no Banco Oracle / } \\
\text { Hang Fire }\end{array}$ & Diário \\
\hline
\end{tabular}

Quadro 1 - Consultas disponíveis no Portal da Transparência do ES

No estudo em que Parker et al. (2006) desenvolveram, foi constatado que um modelo de qualidade da informação para aplicação em portal de transparência na web, deve apresentar algumas das seguintes dimensões, a seguir: acessibilidade, atualidade, exatidão, relevância, credibilidade, objetividade, completeza, representação, fonte, compreensividade.

Além disso Parker et al. (2006) fornecem direções claras e mensuráveis, resumindo as dimensões apresentadas por outros autores para avaliação da qualidade das informações. 
A Acessibilidade (Accessibility) refere-se ao quão facilmente disponíveis estão as informações no site e para download; a Acurácia (Accuracy) refere-se a quão confiáveis e corretas estão as informações na página web; a Adequação (Appropriateness) refere-se a quão adequado é o conteúdo, de acordo, com as necessidades dos visitantes da páginas web; a Credibilidade (Believability) ao quão verdadeiro e credível é o conteúdo na página; a Integridade (Completeness) em relação a falta de dados no conteúdo da web e se são suficiente; A Consistência (Consistency) ao fato de as páginas web serem apresentadas no mesmo formato; a Facilidade de Manipulação (Ease of Manipulation), ou seja, o quão fácil de manipular é o conteúdo da página; Livre de erro (Free-of-Error) refere-se ao fato de as informações sobre a página web serem corretas e confiáveis, ou seja, livre de erros; a Objetividade (Objectivity) refere-se ao quão imparcial e sem preconceitos é o conteúdo da página $W e b$; a Relevância (Relevancy) refere-se a análise do quão aplicável, útil e relevante é o conteúdo da página $W e b$; a Representação (Representation) do quão legível, consistente e estruturado formalmente é o conteúdo da página $W e b$; a Reputação (Reputation) da informação na página da web é altamente considerada no que diz respeito ao seu conteúdo. A Segurança (Security), ou seja, em que medida o acesso à página da Web é restrito de forma adequada para manter a sua segurança; a Velocidade (Speed) de recuperação ou para download do conteúdo de páginas web; a Pontualidade (Timeliness) com a qual o conteúdo da página Web é atualizado; a Compreensibilidade (Understandability) se o conteúdo da página $W e b$ é facilmente compreendido; e, o Valor acrescentado (Valueadded), ou seja, se as informações sobre a página são benéficas e fornecerem vantagens na sua utilização [Parker et al., 2006].

Em um trabalho correlato, Cappelli (2009) propõe a utilização de um Softgoal Interdependency Graph (SIG) [Chung et al., 2000] para representar o conceito de transparência organizacional, contendo algumas as características desejáveis. Carvalho, Cappelli e Santoro (2017), por sua vez, utilizam o SIG de transparência proposto por Cappelli (2009) para sugerir uma abordagem de avaliação de transparência em sites. As características do SIG de transparência de Cappelli (2009) são relacionadas a seguir:

- Acessibilidade, que é subdividida em portabilidade (capaz de ser utilizado em diversos ambientes), disponibilidade (capaz de aceitar solicitações), e publicidade (capaz de ser conhecido);

- Usabilidade, que é subdividida em uniformidade (capaz de manter uma única forma), intuitividade (capaz de ser utilizado sem aprendizado prévio), simplicidade (capaz de não apresentar dificuldades ou obstáculos), amigabilidade (uso fácil), operabilidade (capaz de ser executado), e desempenho (capaz de operar adequadamente).

- Informativo, que é subdividida em clareza (capaz de prover informações com nitidez), acurácia (ausência de erros), completeza (capacidade de não faltar nada do que pode ou deve ter), corretude (capacidade de ser isento de erros), consistência (capacidade de resultar aproximadamente em várias medições de um mesmo item), integridade (capacidade de ser preciso e rigoroso), comparabilidade (capacidade de ser confrontado com outro para lhe determinar diferença, semelhança ou relação), e atualidade (refletir a última atualização ou mudança). 
- Entendimento, que é subdividida em compositividade (capacidade de construir e formar a partir de diferentes partes), concisão (capacidade de ser resumido), divisibilidade (capacidade de ser particionado), e adaptabilidade (capacidade de mudar de acordo com as circunstâncias e necessidade).

- Auditabilidade, que é subdividida em explicável (capaz de explicar dados, procedimentos e regras), rastreabilidade (capaz de seguir o desenvolvimento de um processo ou a construção de uma informação, suas mudanças e justificativas), verificabilidade (capaz de ser legitimado, identificar se o que está sendo feito é o que deve ser feito), validade (capaz de ser testado por experimentação ou observação, identificar se o que está sendo feito é correto), e controlabilidade (capaz de ser controlado).

\section{Monitoramento Automático de Informações de Livre Acesso - MAILA}

Devido à ampliação de informações que vem ocorrendo no Portal da Transparência do Estado do Espírito Santo, e da necessidade de mantê-lo atualizado aos cidadãos, surgiu a necessidade de se criar um robô para auxiliar o processo de análise dos dados, contribuindo para melhorar a qualidade das informações disponíveis aos cidadãos.

"No que toca a organização ou gestão da informação, a quantidade de dados e informações disponíveis tem levado as organizações a implementarem ações para a otimizar a coleta, o tratamento, a localização, análise das informações e avaliação" [Queyras and Quoniam, 2006, p.81].

O desenvolvimento do robô MAILA iniciou no ano de 2014, especificamente para controlar os dados de despesa, pois, à época, ocorriam diversos problemas tanto na extração quanto na atualização dessas informações no portal da transparência. Com o passar dos anos, devido à ampliação das informações disponíveis ao público, houve a necessidade também de se ampliar as validações.

O robô MAILA foi construído em PowerShell, que é uma interface de linha de comando para acessar os serviços dos sistemas operacionais da Microsoft, onde podem ser elaborados scripts sobre a plataforma .NET [Júnior and Gomes, 2013]. O robô, atualmente, está dividido em 3 seções específicas: leitura dos dados; análise dos dados; comunicação do resultado.

\section{Leitura dos dados:}

Com a reestruturação do portal da transparência, as consultas foram desenvolvidas para exibir o valor consolidado e a data em que ocorreu a última atualização dos dados, em formato HTML, permitindo que o script realize a leitura dessas informações. Exemplo: Na consulta de licitações, em seu cabeçalho, são exibidas a quantidade de licitações e o valor gasto no ano; na consulta de despesa, aparecem os valores empenhado, liquidado e pago realizados no ano; na consulta do programa social bolsa capixaba, aparecem a quantidade e o valor pago aos beneficiários no ano etc. 
2. Análise dos dados:

$\mathrm{Na}$ etapa anterior, os valores consolidados disponíveis em cada consulta, juntamente com a data em que ocorreu a última atualização, são armazenados em uma planilha para serem analisados. Com a leitura desses dados, é possível verificar se as informações estão disponíveis para acesso ao público, analisar a tendência de crescimento ou redução com base no histórico e identificar possíveis anomalias, causadas por erros de implementação dos sistemas ou por falhas dos usuários. A análise da tendência de crescimento ou redução, e a identificação das anomalias, é feita mediante comparação dos dados históricos que estão armazenados na planilha. Por exemplo, se determinada informação apresenta histórico de crescimento diário de $5 \% \mathrm{e}$, em determinado dia, cresce $10 \%$, trata-se de um indício de anomalia. O Quadro 2, a seguir, demonstra os problemas identificados pelo robô MAILA, como são apresentados aos usuários e possíveis causas:

\begin{tabular}{|c|c|c|}
\hline Tipo do problema & $\begin{array}{c}\text { Como é apresentado } \\
\text { ao usuário }\end{array}$ & Possíveis causas \\
\hline \multirow{5}{*}{$\begin{array}{l}\text { Informações } \\
\text { indisponíveis para } \\
\text { acesso }\end{array}$} & \multirow{5}{*}{$\begin{array}{l}\text { - as informações não } \\
\text { são exibidas }\end{array}$} & $\begin{array}{l}\text { Sistema de origem está em manutenção e se } \\
\text { encontra off-line }\end{array}$ \\
\hline & & $\begin{array}{l}\text { Sistema de origem sofreu mudança na estrutura } \\
\text { de dados }\end{array}$ \\
\hline & & $\begin{array}{l}\text { Falha na ferramenta de integração que transfere } \\
\text { os dados. }\end{array}$ \\
\hline & & $\begin{array}{l}\text { Falha na infraestrutura que garante a conexão } \\
\text { entre os ambientes }\end{array}$ \\
\hline & & Falha na indexação dos dados (Elastic Search) \\
\hline \multirow[b]{2}{*}{$\begin{array}{l}\text { Informações } \\
\text { desatualizadas }\end{array}$} & \multirow[b]{2}{*}{$\begin{array}{l}\text { - as informações são } \\
\text { apresentadas de forma } \\
\text { desatualizadas }\end{array}$} & Todos as causas listadas no item acima \\
\hline & & $\begin{array}{l}\text { Falha do usuário em não atualizar as } \\
\text { informações na data prevista (esquecimento, } \\
\text { férias, mudança de equipe, ocupado com outras } \\
\text { atividades etc) }\end{array}$ \\
\hline \multirow{3}{*}{$\begin{array}{l}\text { Baixo desempenho } \\
\text { na consulta por } \\
\text { informações }\end{array}$} & \multirow{3}{*}{$\begin{array}{l}\text { - as informações não } \\
\text { são exibidas devido ao } \\
\text { tempo de espera } \\
\text { exceder o timeout do } \\
\text { servidor web }\end{array}$} & Problema de infraestrutura \\
\hline & & Problema de banco de dados \\
\hline & & Problema no código fonte \\
\hline \multirow{4}{*}{$\begin{array}{l}\text { Informações } \\
\text { incorretas } \\
\text { (anomalias) }\end{array}$} & \multirow{4}{*}{$\begin{array}{l}\text { - as informações são } \\
\text { apresentadas com } \\
\text { valores incorretos }\end{array}$} & Importação dos dados incompleta \\
\hline & & Indexação dos dados incompleta \\
\hline & & Duplicação dos dados \\
\hline & & $\begin{array}{l}\text { Falha de controle nos sistemas de origem (ex.: } \\
\text { campo numérico que aceita valores sem } \\
\text { nenhuma restrição) }\end{array}$ \\
\hline
\end{tabular}

Quadro 2 - Problemas identificados pelo robô MAILA, como são apresentados aos usuários e possíveis causas 
3. comunicação do resultado:

O resultado é comunicado aos gestores do Portal da Transparência pelo e-mail corporativo (transparencia@secont.es.gov.br) e registrado em planilha para análise e acompanhamento futuros. Os resultados comunicados também são utilizados pela equipe de gestão para ajudar na identificação de problemas no Portal e nos sistemas fonte dos dados.

\section{Melhoria da qualidade da informação}

Conforme mencionado anteriormente, o robô MAILA é uma ferramenta que contribui para analisar dados do portal da transparência em curto espaço de tempo, tarefa que levaria algumas horas se fosse realizada manualmente.

Atualmente, o Portal da Transparência possui 44 (quarenta e quatro) consultas principais, as quais exibem informações em formato HTML, arquivos para download no formato PDF/XLSX e links para outras páginas. São 30 (trinta) as consultas que são verificáveis, ou seja, que não são links para outras páginas, e, destas, 27 (vinte e sete) são monitoradas pelo robô MAILA, o que equivale a uma abrangência de $90 \%$ das consultas verificáveis no Portal.

Esse procedimento automatizado permite que os gestores tomem conhecimento e consigam agir de forma rápida, muitas vezes, evitando que o problema chegue aos usuários finais.

Ressaltamos que os usuários do Portal da Transparência, cidadãos, estudantes e outros profissionais, dificilmente teriam o discernimento de identificar se o valor apresentado, na cifra de milhões ou bilhões de reais, estão ou não corretos. Informações apresentadas incorretamente, podem estar sendo utilizadas por centenas de pessoas diariamente.

Por isso, o gestor público deve agir rapidamente e garantir a qualidade da informação disponibilizada na internet. No Quadro 03 fazemos uma relação entre os tipos de problemas identificados pelo robô MAILA, com as características de qualidade relatadas por Parker et al. (2006) e com as características do SIG de transparência de Cappelli (2009), que estão momentaneamente ausentes no Portal da Transparência. Cabe ressaltar que nem todas as características presentes no SIG de transparência de Cappelli (2009), que são utilizadas por Carvalho, Cappelli e Santoro (2017) na abordagem de avaliação de transparência em sites, estão relacionadas com a qualidade da informação presente no Portal da Transparência.

Além disso, apesar de algumas características do SIG de transparência em sites estarem presentes no Portal da Transparência, por exemplo, a característica "explicável", de Capelli (2009), uma vez que o Portal possui manual, help online e perguntas frequentes, tais características não estão sendo abordadas neste trabalho, uma vez que não se alteram com frequência e são difíceis de serem analisadas pelo robô MAILA.

\begin{tabular}{|c|c|c|}
\hline $\begin{array}{c}\text { Tipo de problema identificado } \\
\text { pelo robô MAILA }\end{array}$ & $\begin{array}{c}\text { Características } \\
\text { (Parker } \text { et } \text { al, 2006) }\end{array}$ & $\begin{array}{c}\text { Características } \\
\text { (Cappelli, 2009) }\end{array}$ \\
\hline Informações estão indisponíveis & Acessibilidade & Disponibilidade \\
\hline
\end{tabular}




\begin{tabular}{|l|l|l|}
\hline para acesso & & \\
\hline Informações desatualizadas & $\begin{array}{l}\text { Acurácia; Credibilidade; } \\
\text { Pontualidade }\end{array}$ & Atualidade \\
\hline $\begin{array}{l}\text { Baixo desempenho na consulta } \\
\text { por informações }\end{array}$ & Velocidade & Desempenho \\
\hline $\begin{array}{l}\text { Informações incorretas } \\
\text { (anomalias) }\end{array}$ & $\begin{array}{l}\text { Acurácia; Credibilidade; } \\
\text { Livre de erro }\end{array}$ & $\begin{array}{l}\text { Consistência; Corretude; } \\
\text { Integridade }\end{array}$ \\
\hline
\end{tabular}

Quadro 3 - Relação entre os tipos de problemas identificados pelo robô MAILA com as características de qualidade de Parker et al. (2006) e Cappelli (2009)

\section{Benefícios e Resultados}

Automatizar o processo de análise dos dados do portal da transparência resulta em diversos benefícios, destacamos os principais:

- redução do tempo de análise dos dados;

- maior exatidão para analisar dados e encontrar anomalias;

- baixo investimento para realizar manutenções no código fonte;

- monitoramento contínuo, mesmo na ausência dos responsáveis;

- a execução do script pode ser feita diversas vezes ao dia;

- maior confiabilidade das informações para os usuários;

Como resultado principal, podemos citar a identificação de anomalias nos dados, ocasionados devido à falta de controle dos sistemas que processam e armazenam as informações.

Os exemplos a seguir foram identificados pelo robô MAILA por meio de uma análise histórica dos dados, pois, nestes casos, houve um aumento do valor acima da média esperada:

- Em julho de 2017, uma licitação na modalidade de pregão eletrônico, referente à compra de materiais de limpeza, foi publicada no Portal da Transparência com o valor de quase $\mathrm{R} \$ 9$ bilhões (nove bilhões) de reais. O gestor responsável foi alertado e a inspeção detectou uma falha no sistema de compras governamentais.

- Em agosto de 2017, os valores de despesa, referentes ao empenho, liquidação e pagamento, foram duplicados no Portal da Transparência. A equipe técnica foi acionada e a inspeção detectou uma falha no carregamento dos dados de despesa.

- Em outubro de 2017, um contrato no valor de R\$ 62 bilhões (sessenta e dois bilhões) de reais foi apresentado no Portal da Transparência. O gestor responsável foi alertado e a inspeção detectou que o usuário informou o CNPJ da empresa no campo valor do contrato. A informação foi corrigida no sistema de compras governamentais. 
Outros casos identificados pelo robô estão relacionados à desatualização das informações, e suas principais causas são: mudança de ambiente tecnológico, ausência do responsável, mudança de equipe no setor e manutenção do sistema de origem dos dados.

\section{Conclusão}

A transparência é um bom antídoto contra a corrupção, pois incentiva os gestores públicos a serem mais responsáveis em sua atuação e permite que a sociedade, de posse das informações, controle a ação dos governantes e fiscalize a aplicação do dinheiro público.

Manter a qualidade das informações disponíveis aos cidadãos é essencial para alcançarmos a finalidade da transparência pública, por isso, a ampliação de informações nos portais deve ser acompanhada de um monitoramento contínuo dos gestores públicos, para tanto, se faz necessário a utilização de ferramentas para auxiliar a análise dos dados, com o objetivo de realizar as devidas correções em curto espaço de tempo.

A utilização do robô MAILA, para monitorar as informações que são publicadas no Portal da Transparência, tem permitido que o Governo do Estado do Espírito Santo disponibilize informações públicas com qualidade e atualizadas para a sociedade com menor gasto de tempo e de recursos.

Entretanto, algumas limitações foram identificadas, como a operacionalização do robô para analisar dados que estão em outros sítios institucionais ou para verificar a qualidade de informações que não se alteram com frequência e são difíceis de serem identificadas. Como exemplo, podemos citar a característica "explicável”, de Capelli (2009), uma vez que o Portal possui manual, help online e perguntas frequentes, que não se alteram com frequência e, quando são alteradas, são difíceis de serem analisadas pelo robô MAILA, pois são informações que abordam conceitos que dependem de análise de um especialista.

Finalizando, externamos as perspectivas futuras de evoluir o robô MAILA, incluindo novas funcionalidades, tais como:

- Confrontar os dados do diário oficial com o portal da transparência, para identificar informações que não estão nos sistemas de governo e, consequentemente, não estão disponíveis aos cidadãos pelo portal da transparência.

- Aumentar a granularidade na verificação dos dados, identificando se o gasto de um elemento de despesa ocorreu variação relevante, contribuindo, assim, para futuras inspeções e auditorias.

\section{Referências}

Brasil. (2000) "Lei Complementar $\mathrm{n}^{\circ}$ 101, de 4 de maio de 2000". Disponível em: http://www.planalto.gov.br/ccivil_03/leis/lcp/lcp101.htm. Acesso em: 28/03/2018.

Brasil. (2009) "Lei Complementar $\mathrm{n}^{\circ}$ 131, de 27 de maio de 2009". Disponível em: http://www.planalto.gov.br/CCivil_03/leis/LCP/Lcp131.htm. Acesso em: 28/03/2018.

Brasil. (2011) "Lei $\mathrm{n}^{\mathrm{o}}$ 12.527, de 18 de novembro de 2011". Disponível em: http://www.planalto.gov.br/ccivil_03/_ato2011-2014/2011/lei/112527.htm. Acesso em: 28/03/2017. 
Carvalho, L. P., Cappelli, C., and Santoro, F. M. (2017) "Transparência de Software Centrada na Análise de Sites." In Anais do WTRANS17 - Workshop de Transparência em Sistemas, São Paulo, Brasil, Julho 3, 2017.

Cappelli, C. (2009) "Uma Abordagem para Transparência em Processos Organizacionais Utilizando Aspectos". Tese de Doutorado. Pontifícia Universidade Católica do Rio de Janeiro. Rio de Janeiro.

CGU - Controladoria Geral da União. (2018). Transparência Pública. Disponível em: http://www.transparencia.gov.br/sobre. Acesso em 26 de março de 2018.

Chung, L., Nixon, B. A., Yu, E., Mylopoulos, J. (2000) "Non-Functional Requirements in Software Engineering." Kluwer Academic Publishers, Londres

Dabla-Norris, Era et al. (2010). Budget institutions and fiscal performance in low-income countries. IMF working papers, 1-56

Gollwitzer, S. et al. (2010). Budget Institutions and Fiscal Performance in Low-Income Countries. International Monetary Fund.

Júnior, L. C. B. and Gomes, J. C. P. (2013) "Sistema Repositório Wiki de Scripts”. Centro Federal de Educação Tecnológica Celso Suckow da Fonseca, Rio de Janeiro.

Massariol, F.; Louzada, F. R.; Alves, L. F. M. (2017) "Modernização e ampliação do portal da transparência do Espírito Santo: Pensado pelo cidadão para o cidadão". In: X Congresso CONSAD de Gestão Pública, 2017, Brasília/DF.

Parker, M. B., Moleshe, V., De la Harpe, R., and Wills, G. B. (2006). "An evaluation of Information quality frameworks for the World Wide Web".

Queyras, Joachim; Quoniam, Luc. Inteligência competitiva (IC). In: TARAPANOFF, K.(Org.). Inteligência, informação e conhecimento em corporações. Brasília: IBICT, UNESCO, p. 73-97, 2006

Reis, T. (2013). Algoritmo rastreador Web especialista nuclear. Tese de Doutorado. Universidade de São Paulo. São Paulo. 